\title{
On the transient nature of localized pipe flow turbulence
}

\author{
MARC AVILA ${ }^{1}$, ASHLEY P. WILLIS \\ AND BJ ÖRN HOF ${ }^{1}$ \\ ${ }^{1}$ Max Planck Institute for Dynamics and Self-Organization, 37073 Göttingen, Germany \\ ${ }^{2}$ Laboratoire d'Hydrodynamique, École Polytechnique, 91128 Palaiseau, France
}

(Received 11 August 2018)

The onset of shear flow turbulence is characterized by turbulent patches bounded by regions of laminar flow. At low Reynolds numbers localized turbulence relaminarises, raising the question of whether it is transient in nature or it becomes sustained at a critical threshold. We present extensive numerical simulations and a detailed statistical analysis of the data, in order to shed light on the sources of the discrepancies present in the literature. The results are in excellent quantitative agreement with recent experiments and show that the turbulent lifetimes increase super-exponentially with Reynolds number. In addition, we provide evidence for a lower bound below which there are no meta-stable characteristics of the transients, i.e. the relaminarisation process is no longer memoryless.

\section{Introduction}

The development of turbulence in shear flows poses a challenge of great theoretical and practical relevance (Grossmann 2000; Eckhardt et al. 2007). Since the seminal work of Reynolds (1883) on the onset of turbulent fluid motion in a circular pipe, this system has remained a paradigm for transition without linear instability, i.e. subcritical transition. Here, the boundary that separates the laminar flow from turbulence depends not only on the Reynolds number $(R e)$, but also on the characteristics of ambient and external perturbations. In particular, the threshold in perturbation amplitude that must be exceeded to trigger transition scales as $R e^{-\gamma}$, with $\gamma \in[1,2]$ depending on the perturbation details (Hof, Juel \& Mullin 2003; Peixinho \& Mullin 2007; Mellibovsky \& Meseguer 2009). Thus, if great care is taken to minimize all sources of disturbances, the flow can be kept laminar up to Re as large as $10^{5}$ (Pfenniger 1961).

Brosa (1989) showed that even long times after turbulence is initially triggered, relaminarisation to Hagen-Poiseuille flow may occur. Faisst \& Eckhardt (2004) systematically studied the probability of a turbulent trajectory surviving up to time $t$, given by the survivor function

$$
S(t)=P(\text { flow is turbulent at } T \geq t) .
$$

They concluded that relaminaristaion occurs suddenly and that the process is memoryless, i.e. lifetimes are exponentially distributed with $S(t)=\exp [t / \tau]$, where $\tau$ is the mean turbulent lifetime. This behavior had been previously observed in turbulent relaminarisation experiments in plane Couette flow (Bottin \& Chaté 1998; Bottin et al. 1998). The exponential distribution of lifetimes and sensitive dependence on initial conditions (Darbyshire \& Mullin 1995; Faisst \& Eckhardt 2004) is consistent with the presence of 
a repellor in phase space, a strange saddle (see e.g. Kerswell 2005; Eckhardt 2008). The skeleton of the saddle is then constructed from exact unstable solutions of the NavierStokes equations, and the simplest of such solutions correspond to nonlinear travelling waves, discovered numerically by Faisst \& Eckhardt (2003) and Wedin \& Kerswell (2004). Close visits to such travelling waves in turbulent flows were reported in the experiments of Hof et al. (2004) and simulations by Kerswell \& Tutty (2007). Recently, new classes of travelling waves solutions and relative periodic orbits have also been found (Pringle \& Kerswell 2007; Duguet, Pringle \& Kerswell 2008).

Faisst \& Eckhardt (2004) investigated the scaling of mean turbulent lifetimes as $R e$ is increased and proposed a divergence of reciprocal form $\tau(R e) \propto\left(R e_{c}-R e\right)^{-1}$, indicating that turbulence becomes self-sustained at $R e_{c} \simeq 2250$. However, a limitation of the lifetime study of Faisst \& Eckhardt (2004) was the length of the periodic pipe used in their simulations $(5 D)$, chosen to reduce computational costs. At $R e \lesssim 2400$ the experimentally observed flow regimes consist of localized turbulent structures bounded by regions of laminar flow, so-called puffs (Wygnanski \& Champagne 1973). Puffs have a length of about $20 D$ and are characterized by a sharp turbulent-laminar interface at the trailing edge and a slowly diffused leading edge (see figure 1). Thus, long periodic pipes are required to fully capture their relevant dynamics. The first experimental study to determine lifetimes in pipe flow was performed by Peixinho \& Mullin (2006), who carried out Re reduction experiments following the methodology introduced by Bottin et al. (1998) in plane Couette flow. Using a constant-mass-flux pipe of $750 \mathrm{D}$, they perturbed the laminar profile to generate a puff, then reduced $R e$ and measured the downstream distance at which the puff decayed. Their study also supported a divergence of lifetimes of reciprocal form at $R e_{c}=1750$. An experimental study performed in a gravity-driven pipe by Hof et al. (2006), however, rendered an exponential scaling of $\tau$ with Re, indicating that turbulence may be transient for all finite $R e$.

Willis \& Kerswell (2007) performed numerical simulations of a periodic constant-massflux $50 D$ pipe, which allowed for direct comparison with experiment for the first time. Their results qualitatively supported the critical behavior observed by Peixinho \& Mullin (2006), although the extrapolated critical Reynolds number $R e_{c}=1870$ was larger than the experimentally measured value. More recently, Hof et al. (2008) repeated the experimental study with substantial technical improvements over the setup of Hof et al. (2006). The implementation of accurate temperature control and automatisation of the measurement techniques allowed them to prolong the observational time-span up to 8 orders of magnitude, drastically extending all previous investigations. Their measurements up to $R e=2050$ still exhibited relaminarisation and indicated a faster than exponential (but non-diverging) scaling of lifetimes with $R e$, providing evidence that puff turbulence is of transient nature. While the data of Willis \& Kerswell (2007) was incompatible with an exponential scaling, good agreement with the super-exponential scaling of Hof et al. (2008) calls for further numerical investigation.

The main goal of this paper is to provide a comprehensive discussion on the scaling of turbulent lifetimes in pipe-flows, reconciling previous investigations and clarifying the sources of discrepancy. To this aim, the numerical simulations of Willis \& Kerswell (2007) have been extended in $R e$, observation times and sample sizes. This has substantially increased the statistical significance, rendering much reduced and accurate confidence intervals. A detailed account of the methodology and analysis of the data is presented and the influence of initial conditions is examined. The results are in close quantitative agreement with the recent experiments of Hof et al. (2008) in relaminarisation probabilities at each $R e$ investigated, and hence support a super-exponential increase of the lifetimes 


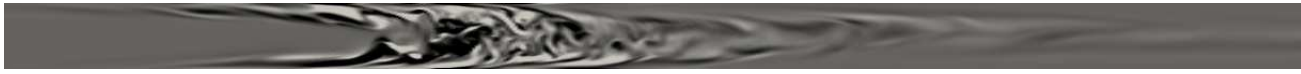

FIgURE 1. Instantaneous stream-wise vorticity distribution in a $(r, z)$-plane of a turbulent puff at $R e=2000$. In the axial direction $20 D$ are shown from a periodic domain of $50 D$.

with $R e$. In addition, it is found that the process is not memoryless for $R e \lesssim 1720$, suggesting a bifurcation event that gives rise to turbulent trajectories.

\section{Numerical Method}

Consider an incompressible viscous fluid which is driven through a pipe of circular cross-section at a constant flow rate. The Reynolds number is defined as $R e=U D / \nu$, where $U$ is the mean flow-speed, $D$ the pipe diameter and $\nu$ the kinematic viscosity of the fluid. It is convenient to scale lengths by $D / 2$ and velocities by $2 U$ in the Navier-Stokes equations, leading to

$$
\partial_{t} \mathbf{u}+(\mathbf{u} \cdot \nabla) \mathbf{u}+\nabla p=\frac{1}{R e} \Delta \mathbf{u}, \quad \nabla \cdot \mathbf{u}=0 .
$$

These are supplied with no-slip boundary conditions at the pipe wall, whereas in the axial direction the pipe is periodic.

The Navier-Stokes equations (2.1) are solved in cylindrical coordinates $(r, \theta, z)$ using the hybrid spectral finite-difference method. This is based on the velocity-potential formulation of Marques (1990), where the difficulties arising from the coupled boundary conditions on the potentials are resolved with the influence-matrix method (Willis \& Kerswell 2009). Variables are expanded in Fourier modes

$$
A=\sum_{k=-K}^{K} \sum_{m=-M}^{M} A_{k, m}(r) \exp [\mathrm{i}(\alpha k z+m \theta)] .
$$

As the variables are real, their Fourier coefficients satisfy the property $A_{k m}=A_{-k,-m}^{\dagger}$, where ${ }^{\dagger}$ denotes the complex conjugate. In the results presented here $K=384$ and $M=24$, corresponding to \pm 384 axial and \pm 24 azimuthal Fourier modes. In the axial direction the wavelength of the pipe is $16 \pi D \simeq 50 D$. This is sufficient to avoid the interaction between leading and trailing edge of the localized structure of a puff (of approximately $20 D$ ) which would arise in short domains. In the radial direction the explicit finite-difference method has been used on 9-point stencils in a grid of $N=40$ points. The time-step was dynamically controlled using information from a predictorcorrector method and was limited to $\Delta t<0.005 \mathrm{D} / \mathrm{U}$. A resolution test on lifetime statistics is shown in the following section. For further details on the formulation, methods and tests see Willis \& Kerswell (2009).

\section{Initial conditions}

In order to generate the initial conditions for the lifetime simulations, a localized disturbance was applied to the laminar Poiseuille flow at $R e=2000$. The disturbance quickly evolved into an 'equilibrium puff' which remained constant in spatial extent while propagating downstream (see figure 1). The puff was evolved for $t \sim 5000 D / U$ and snapshots of the full velocity field were recorded every $10 D / U$, generating a collection of initial conditions. Subsequently, runs at lower $R e$ were performed starting from these initial 
$(a)$

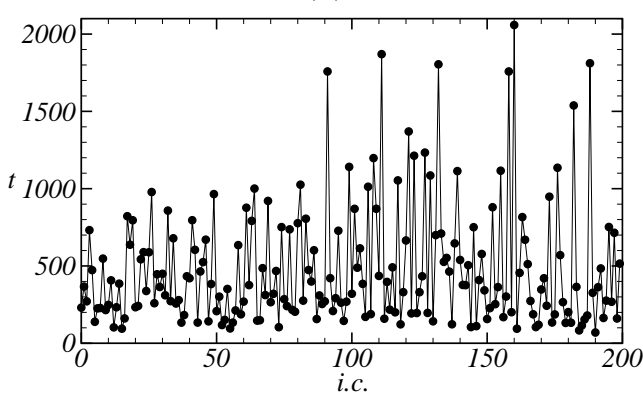

(b)

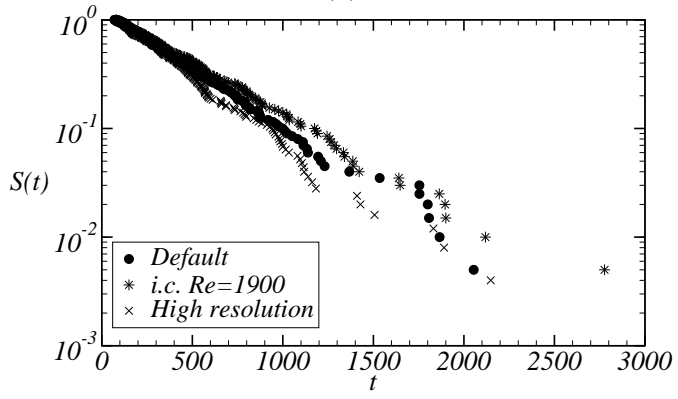

FiguRE 2. (a) Relaminarisation times for reduction simulations from $R e=2000$ to $R e=1860$ as a function of initial condition. (b) Survivor function at $R e=1860$ from the data in (a) (circles), initial conditions at $R e=1900$ (stars) and high numerical resolution (crosses).

conditions and were monitored until the flow relaminarised. The criterion for relaminarisation was that the energy of the axially dependent modes drop below $0.005 U^{2} D^{3}$, at which point turbulent motions had decayed beyond recovery.

Figure 2(a) shows relaminarisation times following a reduction of the Reynolds number to $R e=1860$ as a function of initial condition. The times from here onwards are shown in $D / U$ units. The large fluctuations in the data indicate that consecutive initial conditions result in uncorrelated lifetimes. From these data, the corresponding survivor function $S(t)$ has been obtained and is plotted as circles in the logarithmic scale of figure $2(b)$. The approximately constant slope of $S(t)$ implies the memoryless nature of the relaminarisation process. To verify the numerical resolution, the number of modes in the azimuthal and axial directions was increased by $33 \%$ and the number of finite-difference points by $25 \%$. The number of degrees of freedom is greater than 2.2 times that of the default resolution. The results are plotted in figure $2(b)$ as crosses and are within statistical certainty of the default prediction (see inset of figure 5). Thus, we conclude that the turbulent puffs are accurately resolved.

The impact of the mechanism to initiate the turbulent state on the relaminarisation probabilities has been object of much debate in the literature (Willis \& Kerswell 2007; Schneider \& Eckhardt 2008; de Lozar \& Hof 2009). Indeed, the reduction procedure of Peixinho \& Mullin (2006) was introduced as an attempt to minimise the effect of initial conditions in lifetime measurements. Robustness of the survivor function has been examined here by repeating the lifetime simulations at $R e=1860$ but using three additional sets of initial conditions, each of them consisting of 200 velocity field snapshots. First, initial conditions of a puff simulation at $R e=2200$ were used, representing a much greater initial change of the $R e$ over the default reduction from $R e=2000$. This was again repeated but from a puff simulation at $R e=1925$. Finally, following Willis \& Kerswell (2007) randomly selected velocity snapshots of several puffs at $R e=1900$ were used. The stars in figure $2(b)$ show the results of the reduction from $R e=1900$, whereas results from $R e=2200$ and 1925 are not shown to avoid crowding the figure. All three additional cases render characteristic lifetimes within the $95 \%$ confidence interval about the default prediction (see inset of figure 5), higlighting the independence of the lifetime statistics from initial conditions. These results confirm the experimental findings of de Lozar \& Hof (2009), who have demonstrated the invariance of the lifetime distributions by using four different experimental protocols to generate the turbulent puffs. 
(a)

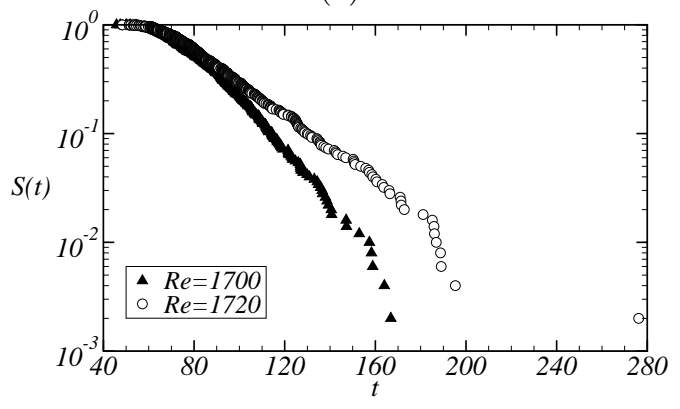

(b)

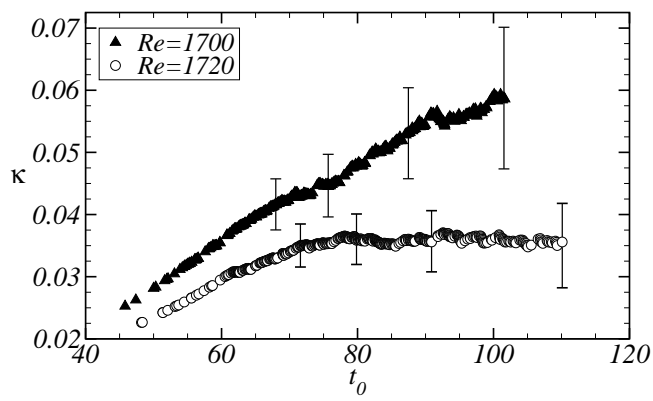

FiguRE 3. (a) Survivor function of turbulent lifetimes at $R e=1720$ (circles) and 1700 (triangles) obtained with 500 simulations each. (b) Escape rates $\kappa=1 / \tau$ as a function of $t_{0}$. The error bars correspond to $95 \%$ confidence intervals around the $\kappa$ predictions.

\section{Statistical analysis of turbulent lifetimes}

An exponential distribution of turbulent lifetimes is one of the main pillars of the strange saddle paradigm for the transition to turbulence in shear flows (Eckhardt 2008). In practice, the distributions are exponential only for $t>t_{0}>0$, where $t_{0}$ depends on the procedure to initiate turbulence and Reynolds number in a non-trivial manner (Schneider \& Eckhardt 2008; de Lozar \& Hof 2009). When Re is suddenly quenched in the reduction experiments, the puff is forced to quickly readjust to the new flow rate and this could cause immediate relaminarisation. In phase space, this is equivalent to a trajectory not having approached the strange saddle. Thus, consistently determining $t_{0}$ constitutes a challenge in lifetime studies of shear flows. The problem stems from the difficulties in discriminating between a trajectory that has shown very short turbulent behavior and one that has directly relaminarised. This is especially delicate in the low $R e$ regime, where a large part of the sample features lifetimes shorter than $t_{0}$.

The hazard function of a lifetime distribution, defined as

$$
h(t)=-\frac{d}{d t} \log (S(t)),
$$

specifies the instantaneous rate of death at time $t$, i.e. $h(t) \delta t$ is the approximate probability of death in $[t, t+\delta t]$ given survival up to time $t$. For an exponential distribution, the hazard function is constant $h(t)=1 / \tau$, where $\tau=\langle t\rangle$ is the mean lifetime of the population. This corresponds to a memoryless process. In the theory of dynamical systems, the escape rate from the saddle is defined as $\kappa=1 / \tau$ (Tél \& Lai 2008).

\subsection{Determining $t_{0}$ : onset of the strange saddle}

Figure $3(a)$ is a logarithmic plot of the lifetime distribution at $R e=1700$ (triangles) and $R e=1720$ (circles). Their corresponding escape rates have been computed by subsequently excluding from the sample puffs that have decayed before a given time $t_{0}$, i.e. $\kappa\left(t_{0}\right)=1 / \tau\left(t_{0}\right)$ with $\tau\left(t_{0}\right)=\left\langle t-t_{0} \mid t>t_{0}\right\rangle$. Figure $3(b)$ shows the resulting values of $\kappa\left(t_{0}\right)$ as a function of cutoff time $t_{0}$. Note that as $t_{0}$ is increased, the size of the sample used to estimate $\kappa$ is progressively reduced from 500 to 100 , resulting in greater error bars. For $R e=1720$, the plot approaches a horizontal line at about $t_{0} \simeq 72$, indicating a constant hazard function and thus exponentially distributed lifetimes. Hence, we conclude that at $R e=1720$ the escape rate of turbulent trajectories from the saddle is $\kappa \simeq 0.035$, whereas trajectories that have decayed before $t_{0} \simeq 72$ cannot be counted as having visited the saddle. Overall $t_{0}$ increases slightly from 72 at $R e=1720$ to 95 at 
$R e=1820$. Meanwhile, the number of trajectories decaying without visiting the saddle decreases as $R e$ increases, and at $R e=1860$ almost all trajectories are attracted to the saddle.

No evidence of exponential behaviour in the lifetimes for $R e<1720$ has been found. This is illustrated by the hazard function at $R e=1700$, plotted as triangles in figure $3(b)$. Despite the initial similarity with $R e=1720$, the escape rate for $R e=1700$ does not settle to a constant value but increases monotonously. This implies that the probabilities of relaminarisation depend on the history of the turbulent trajectory. Similar results have been found for $R e=1580$ and 1640 using 500 simulations in each case. Hence, the data at low $R e<1720$ cannot be used to extrapolate lifetime scaling with $R e$, at least not under the assumption of a memoryless process corresponding to the escape from a strange saddle.

\subsection{Estimation of characteristic turbulent lifetimes}

Characteristic turbulent lifetimes are estimated from an exponential distribution,

$$
S(t)=\exp \left[\left(t-t_{0}\right) / \tau_{\text {true }}\right],
$$

with the sample mean, which is the the Maximum Likelihood Estimator (MLE) of the parameter $\tau_{\text {true }}$. However, the observation time-span is finite in practice, which implies that the data need to be truncated and therefore the sample mean cannot be obtained. A lifetime sample of size $n$ with truncation after $r$ decays is known as censored data of Type-II (Lawless 2003). In this case, the MLE of $\tau_{\text {true }}$ is given by

$$
\tau=\frac{1}{r}\left[\sum_{i=1}^{r} t_{i}+(n-r) t_{r}\right],
$$

where $t_{0}+t_{i}$ is the lifetime of the $i$ th puff to decay, hence $t^{*}=t_{0}+t_{r}$ being the time where the simulations were truncated. The corresponding exact confidence intervals, at level $1-\alpha$, are

$$
\tau_{\text {true }} \in \tau \times\left[2 r / \chi_{2 r, 1-\alpha / 2}^{2}, 2 r / \chi_{2 r, \alpha / 2}^{2}\right],
$$

where $\chi_{m, p}^{2}$ is the $p$ th quantile of the chi-squared distribution with $m$ degrees of freedom. It is worth noting that for uncensored data $(r=n)$, the sample mean is recovered as MLE, and more importantly, that the relative size of the confidence intervals is uniquely determined by the number of runs $r$ that have decayed. In the uncensored case the Central Limit Theorem yields approximate 95\% confidence intervals $\tau \times(1 \pm 1.96 / \sqrt{n})$, and the need for a large number of observations is a consequence of the slow convergence with $n$.

Figure 4(a) shows the survivor function at $R e=1860$ for the first 50 runs in figure 2(a) and for the full sample $n=200$. The mean lifetimes have been estimated from (4.3) and are plotted as solid and dash lines illustrating the curves $\exp \left[\left(t-t_{0}\right) / \tau\right]$. Although the characteristic lifetime from the sub-sample $n=50$ is shorter, its $95 \%$ confidence interval (4.4), spanned by the shaded area in the figure, includes the result from the full sample.

\section{Lifetime scaling with $R e$ : the transient nature of puff turbulence}

In order to shed light on the discrepancies in scaling of turbulent lifetimes present in the literature, we have extended the Reynolds number range in the numerical simulations up to $R e=1900$. The results are presented in figure $4(b)$, showing survivor functions at several $R e$ in a logarithmic scale. The sample sizes have been subsequently increased until each data-set unambiguously showed the exponential distribution. In particular, 
(a)

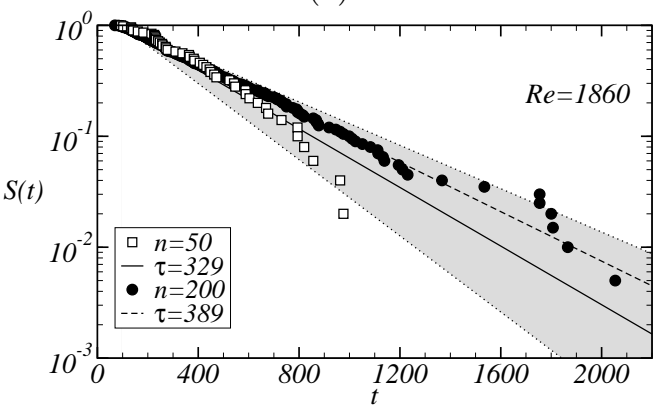

(b)

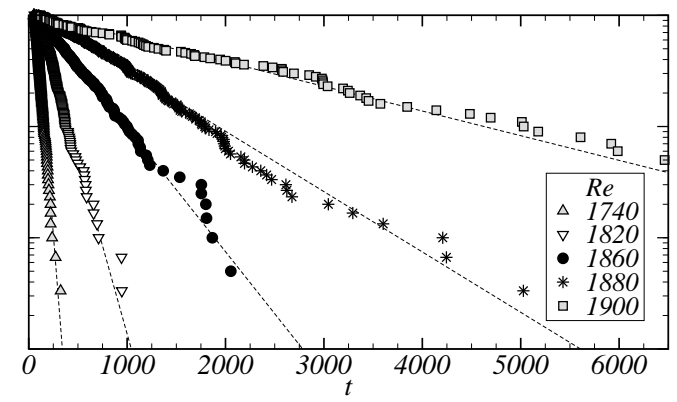

FiguRE 4. (a) Survivor function at $R e=1860$ obtained from sample sizes $n=50$ (squares) and 200 (circles). The solid (dashed) line is the curve $\exp \left[\left(t-t_{0}\right) / \tau\right]$ for $n=50$ (200), where $\tau$ has been estimated from (4.3). The shaded area between the two dotted lines corresponds to the confidence interval (4.4) for $n=50$. (b) Survivor function of turbulent lifetimes at several Reynolds numbers. For clarity, only part of the $R e$ investigated are shown.

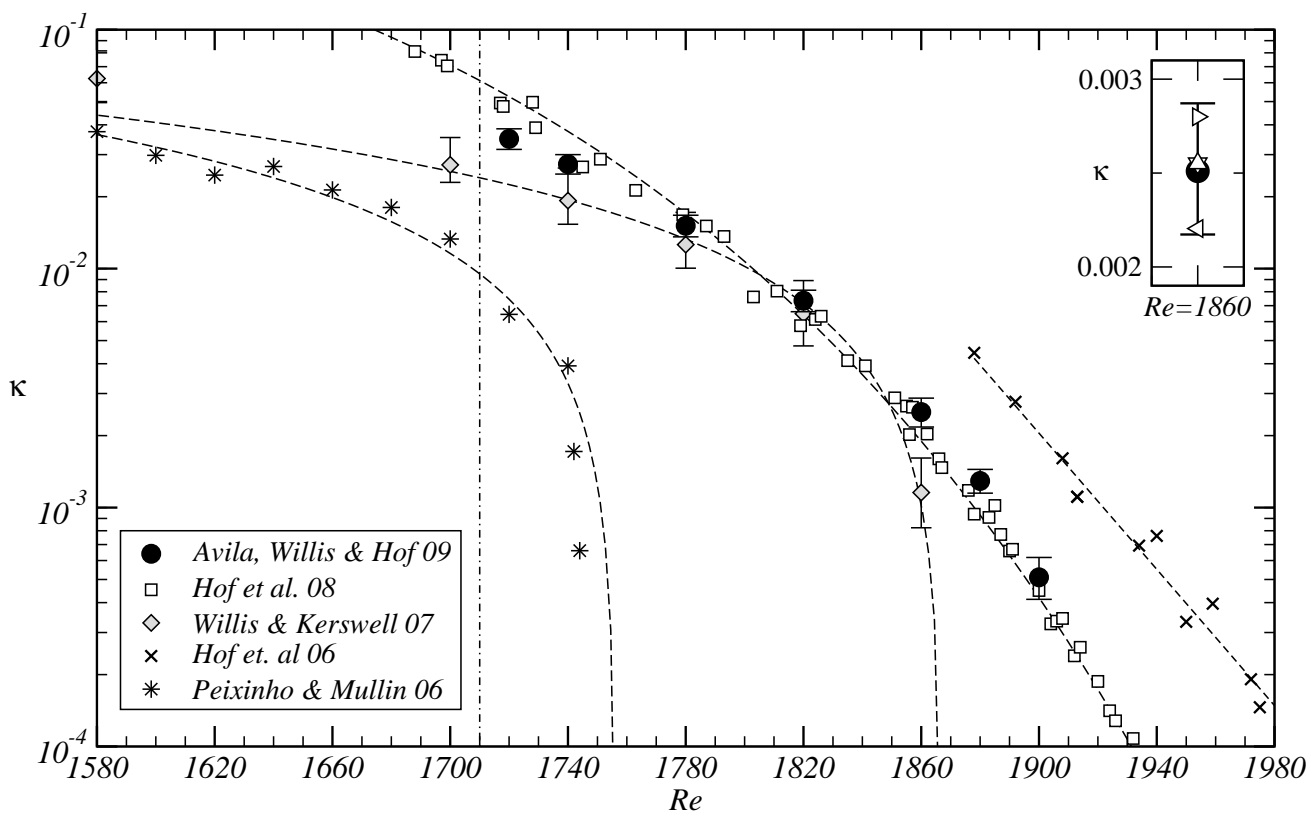

FiguRE 5. Escape rate $\kappa$ scaling with $R e$ as obtained from the mean lifetimes of the survivor functions in figure 4 (shown in black circles). The vertical line marks the approximate onset of the strange saddle. The inset shows the escape rate at $R e=1860$ : default reduction from $R e=2000$ (black circle and 95\% confidence interval), reductions from $R e=2200,1925,1900$ $(\triangle, \nabla, \triangleleft)$ and high numerical resolution $(\triangleright)$.

between 200 and 500 simulations were run for $R e \in[1720,1880]$, whereas at $R e=1900$ only 100 cases were simulated due to the computational costs incurred in very long time integrations. In addition, the simulations at $R e=1900$ were stopped when 96 puffs had decayed, leaving only 4 survivors at $t^{*} \simeq 6500$.

From the survivor functions in figure $4(b)$ escape rates $\kappa=1 / \tau$ have been estimated using (4.3) and are shown as black circles in the logarithmic scale of figure 5 . Confidence intervals at the $95 \%$ level, obtained from (4.4), have been plotted and may be regarded as error bars due to sample size. The results of the high resolution runs and reductions from 
$R e=2200,1925$ and 1900 to $R e=1860$ fall within the $95 \%$ confidence interval of the default case and are only shown in the inset to avoid overlapping the figure. Overall, the results are in excellent quantitative agreement with the experimental relaminarisation probabilities of Hof et al. (2008), shown as squares. With increasing Re, $\kappa$ decreases extremely fast, corresponding to a super-exponential increase in turbulent lifetimes. It is worth noting that the measurements of Hof et al. (2008) extend up to Re $=2050$, with their super-exponential fit providing a very good approximation over the full data set.

The agreement with the simulations of Willis \& Kerswell (2007) is very good for $R e=$ $1740,1780,1820$. For $R e \lesssim 1720$ we have shown that the lifetimes are not exponentially distributed (vertical line). The discrepancy of the point at $R e=1860$ is attributed mainly to statistical uncertainty. There $\tau_{\text {true }}$ was estimated with 40 simulations, of which only 28 had decayed when truncated at $t^{*} \simeq 1000$. The upper end of their confidence interval is close to the experimental value of Hof et al. (2008) and the present computations, although there is still a small difference. We remark, for example, that had the 40 initial conditions $i \in[90,130]$ of figure $2(a)$ been used here to estimate $\tau_{\text {true }}$, the result would be compatible with the estimate provided by Willis \& Kerswell (2007). The escape rates and confidence intervals shown in figure 5 are those given in their re-analysis (Willis \& Kerswell 2008), using bootstrapping. Using the method in $\S 4.2$ on their original data shifts the point at $R e=1860$ by only $10 \%$ in the direction of the current data set, with a $10 \%$ larger confidence interval than that shown.

The experiments of Hof et al. (2006) appear to be shifted by $2.5 \%$ in Re with respect to the numerical simulations and later experiments of Hof et al. (2008). Their exponential fit, however, provides a good approximation of the slope of $\kappa$ over 2 orders of magnitude. Similarly, applying a 7\% shift in Re, the data of Peixinho \& Mullin (2006) overlaps for most of the range. Significant differences between their results and the results presented here, then remain only for their two highest Re. A procedural difference is the manner in which the puffs were generated. It has been shown here and by de Lozar \& Hof (2009), however, that the initial condition has no influence other than on $t_{0}$. A more likely source of difference is the difficulty of inferring the lifetime from the data. At high $R e$, a short pipe implies very few relaminarisations and therefore a very large range of possible $\tau$ according to (4.3). For example, at $R e=1745$ Peixinho \& Mullin (2006) obtained a mean lifetime of $\tau=1515$. For an observation distance of $500 D$ after reducing $R e$, this corresponds to $r \leq 3$ decays out of $n=50$ experimental runs, yielding a confidence interval $\tau_{\text {true }} \times[629,7346]$. Nevertheless, we must acknowledge that Peixinho \& Mullin (2006) were first to experimentally demonstrate the very rapid increase in lifetimes over a small range of Reynolds numbers.

\section{Conclusions}

The transition to turbulence in pipe flow has been recently linked to the presence of a strange saddle in the phase space of the Navier-Stokes equations (see e.g. Eckhardt 2008). One of the predictions of this emerging paradigm is that the turbulent lifetimes, the time prior to escaping the saddle, should be exponentially distributed. We have shown that the distributions are indeed exponential by substantially increasing the sample size and thus reducing statistical uncertainty with respect to previous works. In order to recover the true escape rate, a substantially larger sample size than previously used is necessary.

We have demonstrated that initial conditions from puffs at $R e=2200,2000,1925,1900$ render the same characteristic lifetimes after reduction to $R e=1860$, which verifies that the same turbulent state is visited. Here, contrary to the possibility that the turbulent 
state be difficult to reach when changing the Reynolds number, the number of trajectories which fail to approach the saddle is actually low (small $t_{0}$ ). This reflects structural similarity of the initial condition with the state at the final $R e$.

Interestingly, the onset of the exponential distributions is rather sharp in $R e$ at about $R e \lesssim 1720$. This suggests a bifurcation event that gives rise to the strange saddle. The onset of meta-stable transients has been characterized in plane Couette flow experiments by Bottin \& Chaté (1998) and Bottin et al. (1998), who measured the average turbulent fraction. The change in distributional shape revealed here provides an alternative reliable means of quantifying a lower bound for meta-stable turbulence, and is appropriate when considering single isolated disturbances.

Our results support the view of localised turbulence as a strange repellor, with lifetimes increasing super-exponentially with $R e$ as in the experiments of Hof et al. (2008). A similar scaling has been recently reported by Borrero-Echeverry, Tagg \& Schatz (2009) in Taylor-Couette flow with stationary inner cylinder and previously by Schoepe (2004) in super-fluid turbulence. To our knowledge the quantitative agreement of the presented numerical simulations with experiment is unprecedented in transition studies. It validates the use of periodic boundary conditions and testifies the high demands on numerical resolution and domain sizes which are required to faithfully capture the relevant dynamics of turbulence.

We wish to thank many cited authors for interesting and insightful discussions regarding lifetimes in shear flows. M. Avila and B. Hof are supported by the Max Planck Society. A. P. Willis is supported by the E.C., Marie Curie Fellowship PIEF-GA-2008-219223.

\section{REFERENCES}

Borrero-Echeverry, D., TAgg, R. \& Schatz, M.F. 2009 Transient Turbulence in TaylorCouette Flow. arXiv:0905.0147v1.

Bottin, S. \& Chaté, H. 1998 Statistical analysis of the transition to turbulence in plane Couette flow. Eur. Phys. J. B 6 (1), 143-155.

Bottin, S., Daviaud, F., Manneville, P. \& Dauchot, O. 1998 Discontinuous transition to spatiotemporal intermittency in plane Couette flow. Europhys. Lett. 43 (2), 171-176.

Brosa, U. 1989 Turbulence without strange attractor. J. Stat. Phys. 55 (5), 1303-1312.

Darbyshire, A.G. \& Mullin, T. 1995 Transition to turbulence in constant-mass-flux pipe flow. J. Fluid Mech. 289, 83-114.

Duguet, Y., Pringle, C.C.T. \& Kerswell, R.R. 2008 Relative periodic orbits in transitional pipe flow. Physics of Fluids 20, 114102.

ECKHARDT, B. 2008 Turbulence transition in pipe flow: some open questions. Nonlinearity (London) 21 (1), T1-T11.

Eckhardt, B., Schneider, T.M., Hof, B. \& Westerweel, J. 2007 Turbulence transition in pipe flow. Annu. Rev. Fluid Mech. 39, 447-468.

Faisst, H. \& Eckhardt, B. 2003 Traveling waves in pipe flow. Phys. Rev. Lett. 91 (22), 224502.

Faisst, H. \& Eckhardt, B. 2004 Sensitive dependence on initial conditions in transition to turbulence. J. Fluid Mech. 504, 343-352.

Grossmann, S. 2000 The onset of shear flow turbulence. Rev. Mod. Phys. 72 (2), 603-618.

Hof, B., van Doorne, C.W.H., Westerweel, J., Nieuwstadt, F.T.M., Faisst, H., Eckhardt, B., Wedin, H., Kerswell, R.R. \& Waleffe, F. 2004 Experimental observation of nonlinear traveling waves in turbulent pipe flow. Science 305 (5690), 1594-1598.

Hof, B., Juel, A. \& Mullin, T. 2003 Scaling of the turbulence transition threshold in a pipe. Phys. Rev. Lett. 91 (24), 244502.

Hof, B., De Lozar, A., Kuik, D.J. \& Westerweel, J. 2008 Repeller or Attractor? Selecting the Dynamical Model for the Onset of Turbulence in Pipe Flow. Phys. Rev. Lett. 101 (21), 214501. 
Hof, B., Westerweel, J., Schneider, T. M. \& Eckhardt, B. 2006 Finite lifetime of turbulence in shear flows. Nature (London) 443 (7), 59-62.

Kerswell, R. R. 2005 Recent progress in understanding the transition to turbulence in a pipe. Nonlinearity (London) 18 (6), R17R44.

Kerswell, R. R. \& TutTy, O.R. 2007 Recurrence of travelling waves in transitional pipe flow. J. Fluid Mech. 584, 69-102.

Lawless, J.F. 2003 Statistical Models and Methods for Lifetime Data, 2nd edn. New Jersey: Wiley.

DE LozAR, A. \& Hof, B. 2009 An experimental study of the decay of turbulent puffs in pipe flow. Phil. Trans. R. Soc. A 367 (1888), 589-599.

MARques, F. 1990 On boundary conditions for velocity potentials in confined flows. Application to Couette flow. Phys. Fluids A 2, 729-737.

Mellibovsky, F. \& Meseguer, A. 2009 Critical threshold in pipe flow transition. Phil. Trans. R. Soc. A 367 (1888), 545-560.

Peixinho, J. \& Mullin, T. 2006 Decay of Turbulence in Pipe Flow. Phys. Rev. Lett. 96 (9), 094501.

Peixinho, J. \& Mullin, T. 2007 Finite-amplitude thresholds for transition in pipe flow. $J$. Fluid Mech. 582, 169-178.

Pfenniger, W. 1961 Transition in the inlet length of tubes at high Reynolds numbers. In Boundary Layer and flow control (ed. G. V. Lachman), pp. 970-980. New York: Pergamon.

Pringle, C.C.T. \& Kerswell, R.R. 2007 Asymmetric, helical, and mirror-symmetric traveling waves in pipe flow. Phys. Rev. Lett. 99, 074502.

Reynolds, O. 1883 An Experimental Investigation of the Circumstances Which Determine Whether the Motion of Water Shall Be Direct or Sinuous, and of the Law of Resistance in Parallel Channels. Proc. R. Soc. London 35, 84-99.

Schneider, T.M. \& ECKhardt, B. 2008 Lifetime statistics in transitional pipe flow. Phys. Rev. E 78 (4), 046310.

Schoepe, W. 2004 Fluctuations and stability of superfluid turbulence at $\mathrm{mK}$ temperatures. Phys. Rev. Lett. 92 (9), 95301.

TÉL, T. \& LAI, Y.C. 2008 Chaotic transients in spatially extended systems. Phys. Rep. 460 (6), 245-275.

Wedin, H. \& Kerswell, R.R. 2004 Exact coherent structures in pipe flow: travelling wave solutions. J. Fluid Mech. 508, 333-371.

Willis, A.P. \& Kerswell, R.R. 2007 Critical Behavior in the Relaminarization of Localized Turbulence in Pipe Flow. Phys. Rev. Lett. 98 (1), 014501.

Willis, A.P. \& Kerswell, R.R. 2008 Reply to Comment on 'Critical behaviour in the relaminarization of localized turbulence in pipe flow'. arXiv:0707.2684.

Willis, A.P. \& Kerswell, R.R. 2009 Turbulent dynamics of pipe flow captured in a reduced model: puff relaminarization and localized 'edge' states. J. Fluid Mech. 619, 213-233.

Wygnanski, I. J. \& Champagne, F. H. 1973 On transition in a pipe. Part 1. The origin of puffs and slugs and the flow in a turbulent slug. J. Fluid Mech. 59 (02), 281-335. 